\title{
Erwerbsminderungsrenten $=$ Armutsrenten. Ein vergessenes soziales Problem?
}

Wer von drohender Altersarmut spricht, der muss auch und vor allem auf die Erwerbsminderungsrenten (EM-Renten) schauen. Die durchschnittlichen Zahlbeträge bei den Erwerbsminderungsrenten sinken seit Jahren und liegen deutlich unter dem Grundsicherungsniveau. Reformen sind dringend erforderlich. Aber welche Faktoren führen zu diesem Abwärtstrend? Was muss getan werden, um hier zu einer Umkehr zu kommen? Eine einfache Lösung gibt es nicht, mehrere Schritte sind erforderlich: Ursachen sind zum einen bei den Leistungseinschnitten im Rentenrecht zu suchen, die revisionsbedürftig sind. Zum anderen ist zu berücksichtigen, dass sich die Erwerbsbiografien von EM-Rentnern als besonders risikoträchtig erweisen. Eine präventiv ansetzende Reformpolitik muss deshalb auch die Verwerfungen auf dem Arbeitsmarkt und in den Beschäftigungsverhältnissen bekämpfen.

GERHARD BÄCKER

\section{Einführung}

Die Frage nach der drohenden Altersarmut sowie die Suche nach Reformen im Alterssicherungssystem stehen - gerade vor dem Hintergrund der jüngsten Bundestagswahl und der Neukonstitution der Bundesregierung - im Mittelpunkt der sozialpolitischen Debatten. Unstrittig ist, dass in bereits absehbarer Zeit die Zahl der Altersrenten wachsen wird, die an oder unterhalb der Armutsgrenze liegen. Denn die Veränderungen und Verwerfungen auf dem Arbeitsmarkt führen dazu, dass immer mehr Arbeitnehmer mit prekären Erwerbsbiografien in den Altersübergang und Rentenbezug kommen und entsprechend nur geringe Rentenanwartschaften bzw. Entgeltpunkte aufweisen. Zugleich wird infolge der gedämpften Rentenanpassung (Riester- und Nachhaltigkeitsfaktor) das Rentenniveau sinken, mit der Konsequenz, dass die weniger werdenden Entgeltpunkte auch noch an Wert verlieren. Um dieser Zangenbewegung entgegenwirken zu können, werden mehr oder weniger weitreichende Rentenreformschritte diskutiert. Die Stichworte lauten „solidarische Mindestrente“, „Garantierente“, „Solidarrente“, „Lebensleistungsrente“.

Bei allen, durchaus gravierenden Unterschieden in den Ausgestaltungsdetails dieser Modelle ist ein Charakteristikum verbindend: Die Rede ist immer nur von Altersrenten, also von Renten, die mit dem Erreichen der jeweiligen Altersgrenzen bezogen werden können. Vergessen oder aus- geklammert wird aber, dass die Rentenversicherung nicht nur Altersrenten, sondern auch Erwerbsminderungsrenten gewährt. Zwar ist in der Tat die häufigste Form des Bezugs einer Rente die Altersrente. Aber die Rente wegen Erwerbsminderung ist keineswegs unbedeutend. So haben im Jahr 2012 gut 179.000 Personen erstmalig eine Erwerbsminderungsrente erhalten, das entspricht 21,6 \% aller Zugänge an Versichertenrenten in diesem Jahr (DRV 2013a).

Dass das Thema der Erwerbsminderungsrenten aus den sozial- und rentenpolitischen Reformdebatten ausgeklammert wird, ist aber nicht nur wegen ihrer Größenordnung unverständlich. Denn empirische Befunde zeigen, dass sich das Risiko niedriger Rentenzahlbeträge vor allem auf die Bezieherinnen und Bezieher einer Erwerbsminderungsrente konzentriert. Wenn also ein Ansteigen von Armut im Alter befürchtet und Reformbedarfe als unumgänglich angesehen werden, dann darf das Thema Erwerbsminderungsrenten nicht unberücksichtigt bleiben. Es gehört vielmehr an die Spitze der sozialpolitischen Agenda. Voraussetzung dafür ist, die Dimensionen, Ausprägungen und Ursachen des Problems zu ermitteln, um auf dieser Grundlage Handlungsalternativen aufzuzeigen. Dies soll in den folgenden Ausführungen geschehen.

Zum besseren Verständnis der Thematik ist es zunächst erforderlich, die Grundlagen des Erwerbsminderungsrechts in Erinnerung zu rufen: Seit der Reform von 2001 gilt ein Versicherter dann als voll erwerbsgemindert und hat Anspruch auf eine volle EM-Rente, wenn er aus gesundheitli- 
chen Gründen auf nicht absehbare Zeit nur noch weniger als drei Stunden pro Tag arbeiten kann. Eine halbe Erwerbsminderungsrente erhalten Erwerbsgeminderte bei einem Restleistungsvermögen auf dem allgemeinen Arbeitsmarkt von drei bis unter sechs Stunden täglich. ${ }^{1}$ Die Rente wegen teilweiser Erwerbsminderung ist deshalb nur halb so hoch wie eine Rente wegen voller Erwerbsminderung, weil die Betroffenen mit dem ihnen verbliebenen Restleistungsvermögen grundsätzlich noch das zur Ergänzung der Rente notwendige Einkommen erarbeiten können. Wer unter den üblichen Bedingungen des allgemeinen Arbeitsmarktes mindestens sechs Stunden pro Tag arbeiten kann, ist also nicht erwerbsgemindert und wird, obwohl eine vollschichtige Tätigkeit (acht Stunden pro Tag) nicht möglich ist, völlig aus dem Leistungsbezug ausgeschlossen. Diese an den täglichen Arbeitsstunden gemessene Abgrenzung findet ihre Parallele im Sozialgesetzbuch, Drittes Buch (SGB III: Arbeitslosenversicherung) und im SGB II (Grundsicherung für Arbeitsuchende): Als erwerbsfähig - und damit potenziell auch arbeitslos - gelten all jene, die für mindestens drei Stunden täglich erwerbstätig sein können. Nur bei Erwerbsfähigkeit können auch Ansprüche aus der Arbeitslosenversicherung oder der Grundsicherung für Arbeitsuchende (Arbeitslosengeld II) geltend gemacht werden.

Anspruchsvoraussetzung für den Bezug einer Erwerbsminderungsrente ist zudem die Erfüllung der allgemeinen Wartezeit von fünf Jahren mit Versicherungszeiten, Beitragsoder Ersatzzeiten. Zudem müssen in den letzten fünf Jahren vor Eintritt der Erwerbsminderung mindestens drei Jahre mit Pflichtbeiträgen belegt sein (vgl. im Detail Bäcker 2012).

Renten wegen verminderter Erwerbsfähigkeit werden längstens bis zum Erreichen des Regelrentenalters gewährt und dann in eine Regelaltersrente umgewandelt. Im statistisch ausgewiesenen Rentenbestand finden sich entsprechend wenige Erwerbsminderungsrentner, da Versichertenrenten ab der Regelaltersgrenze $(65+)$ nur noch als Altersrenten ausgewiesen werden. Dies hat zur Folge, dass bei der nachfolgenden Analyse der Dimensionen und Ursachen von niedrigen EM-Renten ausschließlich auf die Rentenneuzugänge Bezug genommen wird.

\section{Kontinuierlicher Rückgang der Ren- tenhöhe - Analyse der Ursachen}

Eine Auswertung der Erwerbsminderungsrenten zeigt, dass in der Zeitspanne zwischen 2000 und 2011 die durchschnittlichen Zahlbeträge (Nettorenten vor Steuern) der neu zugehenden Erwerbsminderungsrenten kontinuierlich abgesunken sind. Lediglich im Rentenzugang des Jahres 2012 zeigt sich ein schwacher Anstieg. Aus Abbildung 1 lässt sich erkennen, dass die Zahlbeträge sowohl bei den Männern als auch bei den Frauen und sowohl in den alten als auch in den neuen Bundesländern rückläufig sind. Besonders
ABB. 1

\section{Erwerbsminderungsrenten: Durchschnittliche Zahlbeträge 2000 - 2012*}

Angaben in Euro
Männer, Westdeutschland
Frauen, Westdeutschland
Männer, Ostdeutschland
Frauen, Ostdeutschland

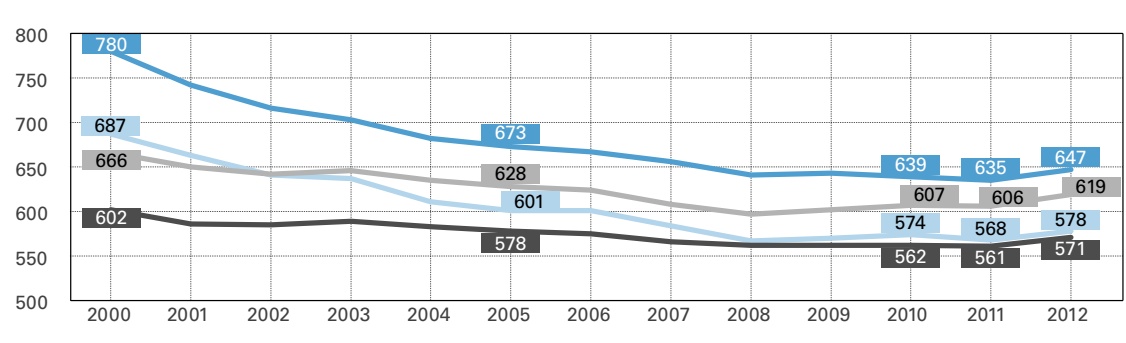

*Männer und Frauen in West- und Ostdeutschland.

Quelle: DRV 2013b, verschiedene Jahrgänge; Berechnungen und Darstellung des Autors. ausgeprägt ist der Rückgang bei den Männern in den alten Bundesländern: von durchschnittlich $780 €$ im Jahr 2000 auf durchschnittlich $647 €$ im Jahr 2012. Noch nicht berücksichtigt ist dabei der Geldwertverlust durch die Preisentwicklung. Welche Faktoren sind für diesen Trend verantwortlich?

\subsection{Entwicklung von Rentenniveau und aktuellem Rentenwert}

Bei der Suche nach den Ursachen für die Abwärtsbewegung muss in einem ersten Schritt die Entwicklung des aktuellen Rentenwerts betrachtet werden, da nach der Rentenformel die Höhe der Erwerbsminderungsrenten (analog zur Berechnung der Altersrenten) durch die Multiplikation der Entgeltpunkte mit dem aktuellen Rentenwert ermittelt wird. In den Jahren seit 2000 ist der aktuelle Rentenwert nur sehr langsam angestiegen und in den Jahren 2004, 2005, 2006 und 2010 aufgrund von sog. „Nullanpassungen“ sogar unverändert geblieben. Diese Entwicklung „drückt“ auf die Höhe der Alters- wie auch der Erwerbsminderungsrenten und ist eine Folge sowohl der schwachen Lohn-

1 Wenn teilweise Erwerbsgeminderte keinen - diesen Zeitvorgaben entsprechenden -Teilzeitarbeitsplatz finden und arbeitslos werden, so muss ihnen (nach entsprechenden Bemühungen der Arbeitsagentur) eine volle Erwerbsminderungsrente gewährt werden. Denn die seit einem Urteil des Bundessozialgerichtes (BSG) von 1976 entwickelte Rechtsprechung zur "konkreten Betrachtungsweise“ berücksichtigt für eine volle Erwerbsminderung nicht allein gesundheitliche Schäden, sondern gleichrangig auch das Fehlen eines geeigneten (Teilzeit)Arbeitsplatzes. Eine volle Erwerbsminderungsrente erhalten deswegen auch teilweise Erwerbsgeminderte, die ihr Restleistungsvermögen wegen eines "verschlossenen Arbeitsmarktes" nicht in Erwerbseinkommen umsetzen können. 
ABB. 2

\section{Erwerbsminderungsrenten: Durchschnittliche Zahlbeträge 2000 - 2012* - nach Anpassung}

Angaben in Euro

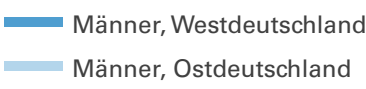

\section{Frauen, Westdeutschland} Frauen, Ostdeutschland

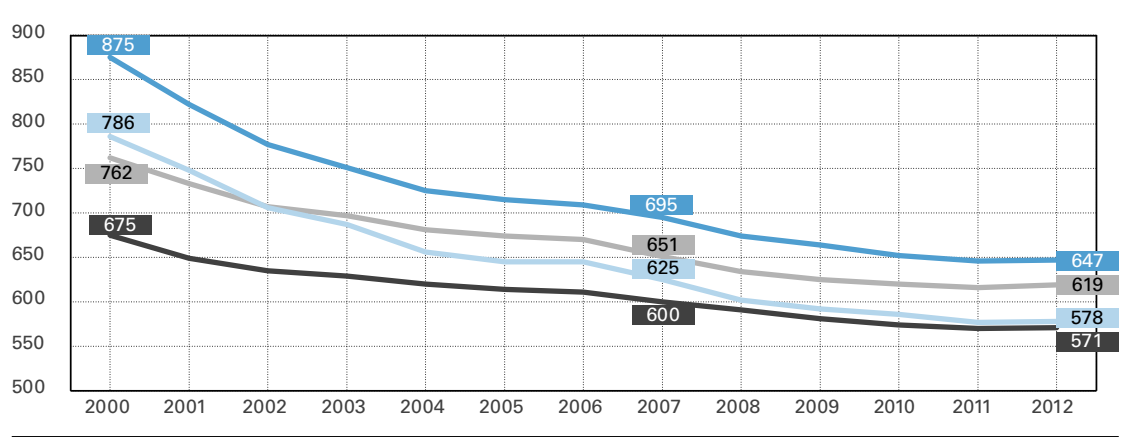

*Männer und Frauen in West- und Ostdeutschland.

entwicklung in den zurückliegenden Jahren als auch der Veränderungen im Rentenanpassungsverfahren in Form des Riester- und Nachhaltigkeitsfaktors. Das Rentenniveau, also das Verhältnis der Einkommensposition der Rentenempfänger gegenüber der Einkommensposition der erwerbstätigen Versicherten, ist gesunken und wird weiter sinken. Allerdings: Trotz des Rückgangs des Rentenniveaus ist der aktuelle Rentenwert (der allerdings nicht mehr die Lohnentwicklung widerspiegelt) noch gestiegen - zwischen 2000 und 2012 in den alten Bundesländern um etwa 13,0 \%. Dabei handelt es sich um Bruttobeträge, die durch die steigenden Beiträge zur Krankenversicherung der Rentner (KVdR) und zur Pflegeversicherung der Rentner (PVdR) noch gemindert werden.

Dieser Anstieg des aktuellen Rentenwerts muss bei dem Vergleich der durchschnittlichen Rentenhöhen im Zugang berücksichtigt werden, da der aktuelle Rentenwert, der für das Jahr 2012 gilt, in die Berechnung einer jeden Rente einfließt und damit auch die vormaligen Zugangsrenten erfasst (vgl. auch Steffen 2013). Das heißt an einem Beispiel konkretisiert: Der Wert der EM-Zugangsrenten aus dem Jahr 2000 liegt im Jahr 2012 um etwa 13 \% höher. Berechnet man also die Zugangsrenten seit 2000 mit dem aktuellen Rentenwert von 2012, um von einer gleichen Referenzgröße auszugehen, dann fällt die Abwärtsbewegung noch weit stärker aus (Abbildung 2). Bei den Männern (West bzw. Ost) sinkt der Zahlbetrag um 26,1 bzw. 18,8 \% und bei den Frauen um 15,4 bzw. $26,5 \%$.

\subsection{Abschläge und ihre Folgen}

Bei einer Inanspruchnahme einer EM-Rente zwei Jahre vor der Regelaltersgrenze wird die Rente, errechnet gemäß der bereits genannten Formel, durch Abschläge bis zu einer maximalen Höhe von 10,8 \% vermindert. Die Abschläge betragen (wie bei vorzeitiger Inanspruchnahme von Altersrenten) $0,3 \%$ pro Monat der Inanspruchnahme, sind aber auf drei Jahre begrenzt. Sie folgen der Zielsetzung, Ausweichreaktionen von einer abschlagsgeminderten vorzeitigen Altersrente für schwerbehinderte Menschen auf eine abschlagsfreie Erwerbsminderungsrente zu vermeiden. Allerdings greifen die Abschläge auch, wenn der Erwerbsminderungsfall weit früher eintritt, z. B. im 40. oder 50. Lebensjahr.

Zwar ist zu berücksichtigen, dass zeitgleich zur Einführung der Abschläge auch die Zurechnungszeiten ${ }^{2}$ verlängert worden sind. Die rentenkürzenden Wirkungen der Abschläge werden dadurch aber nur teilweise ausgeglichen. Die Bundesregierung beziffert den um die Zurechnungszeiten verminderten Verlust durch die Abschläge auf 3,3 \% bei einem Rentenfall bis zum Lebensalter 56 Jahre und 8 Monate (Deutscher Bundestag 2000, S. 24). Der Verlust gegenüber dem alten Recht wird umso höher, je älter die Betroffenen sind. Bei einem erstmaligen Bezug einer Erwerbsminderungsrente mit 60 Jahren, also in einem Alter, $\mathrm{ab}$ dem auch eine vorgezogene Altersrente wegen Schwerbehinderung bezogen werden kann und ein Ausweichverhalten möglich wird, wirken sich dann allein die Abschläge aus.

Schon wenige Jahre nach Einführung dieser Regelung haben die Abschläge eine nachhaltige Wirkung entfaltet: Ab 2004 und seitdem unverändert liegt die Betroffenheit bei über 90 \%, die Zahl der durchschnittlichen Abschlagsmonate beträgt etwa 35 Monate und die Höhe des durchschnittlichen Abschlagsbetrags etwa $78 €$ (DRV 2012). Da damit die Maximalwerte nahezu erreicht sind, spielen die Abschläge für die Fortsetzung des Abwärtstrends bei den EM-Renten nach 2004 keine Rolle mehr.

\subsection{Rückläufige Entgeltpunkte: Folge von Langzeitarbeitslosigkeit und prekärer Beschäftigung}

Nach der Rentenformel spiegeln die Entgeltpunkte die relative Einkommensposition wider, die der Versicherte im Verlauf seiner Versicherungsbiografie im Verhältnis zum Durchschnittsbruttoeinkommen aller Versicherten erreicht hat. Abbildung 3 zeigt, dass bei den Zugängen in Erwerbsminderungsrenten - Männer und Frauen in den alten und neuen Bundesländern - die durchschnittlichen Entgeltpunkte je Versicherungsjahr seit 2010 kontinuierlich gesunken sind. Bis zum Jahr 2011 errechnen sich bei den Männern

2 Um die Versicherungsdauer zu verlängern, werden die Jahre vor dem vollendeten 60. Lebensjahr als Zurechnungszeiten berücksichtigt. Die Rentenberechnung erfolgt so, als hätte der Versicherte bis zum 60. Lebensjahr weiter verdient und Beiträge bezahlt. 
Verluste von 19 \% (West) bzw. 18,3 \% (Ost). Bei den Frauen fällt dieser Trend schwächer als bei den Männern aus, da hier vor allem die Anrechnung von Kindererziehungszeiten zu einer Entlastung führt.

Die rückläufige Entwicklung der Entgeltpunkte lässt sich durch die Veränderungen der Arbeitsverhältnisse und der Erwerbsbiografien erklären, die den Arbeitsmarkt seit Jahren prägen: Die Stichworte lauten: Niedriglöhne, unstete Beschäftigung, Zeiten von Mehrfach- und Langzeitarbeitslosigkeit (Frommert/Himmelreicher 2010; Trischler 2012). Die Erwerbsminderungsrentner sind von diesen Verwerfungen im besonderen Maße betroffen, da sie überproportional häufig ein nur niedriges Qualifikationsniveau aufweisen und (damit im engen Zusammenhang) Tätigkeiten mit hohen körperlichen und/oder psychischen Belastungen ausgeübt haben (Hagen et al. 2011; Rehfeld 2006). Zugleich ist bekannt, dass sich die Arbeitsmarktrisiken auf diese Beschäftigtengruppen konzentrieren (Weber/Weber 2013).

So lässt sich feststellen, dass der Anteil der Versicherten, die im Jahr vor dem Leistungsfall arbeitslos waren bzw. Leistungen nach dem SGB III und SGB II erhielten, seit Jahren steigt. 2011 waren 38,2 \% der Erwerbsminderungsrentner zuvor arbeitslos, darunter zu 29,8 \% mit Bezug von Arbeitslosengeld $\mathrm{II}^{3}$ (Abbildung 4). Bezieht man sich allein auf die Vollrenten, so kommen hier sogar 32,4\% aus dem SGB-IILeistungsbezug und 8,3\% aus dem SGB-III-Leistungsbezug. Demgegenüber sinkt der Anteil jener, die aus einer versicherungspflichtigen Beschäftigung heraus eine Erwerbsminderungsrente erhalten. 2011 standen in dem Jahr vor dem Leistungsfall nur noch $42,7 \%$ in einem versicherungspflichtigen Beschäftigungsverhältnis gegenüber 54,5 \% im Jahr 2000. Im Jahr 2012 scheint sich das Bild zu ändern, da der Anteil derer deutlich sinkt, die zuvor Leistungen nach dem SGB II erhalten haben. Dieser Rückgang ist allerdings allein auf die Veränderung der Rechtslage zurückzuführen: Zeiten des Bezugs von Arbeitslosengeld II sind ab 2011 keine Beitragszeiten mehr, sondern nur noch Anrechnungszeiten. Entsprechend ist 2012 der Anteil der sog. Anrechnungszeitversicherten am Rentenzugang auf 9,5\% gestiegen (DRV 2013b).

Arbeitslosigkeit, und hier insbesondere Langzeitarbeitslosigkeit, stellt damit ein zentrales Eintrittstor in die Erwerbsminderung dar. Die Zusammenhänge lassen sich in zweifacher Hinsicht erklären: Arbeitnehmer mit gesundheitlichen Beeinträchtigungen haben ein besonders hohes Risiko, arbeitslos zu werden und zu bleiben. Zugleich führt ein mehrjähriger Verbleib in der Arbeitslosigkeit zu einer Gefährdung der physischen und vor allem psychischen $\mathrm{Ge}$ sundheit bzw. verstärkt schon vorhandene Einschränkungen (Mika 2013).

Insofern kann es nicht verwundern, dass vor allem die Rentenhöhe nach dem Bezug von Arbeitslosengeld II stark abfällt (Abbildung 5). Die bereits vermutete Sozialstruktur der (Langzeit)Arbeitslosen bestätigt sich hier: Dieser Personenkreis, der 2011 fast ein Drittel der Zugänge an Erwerbsminderungsrenten ausmacht (30 \% der Erwerbs-
ABB. 3

\section{Durchschnittliche Entgeltpunkte je Versicherungsjahr der Neuzugänge von Erwerbsminderungsrenten 2000 - 2011*}

Männer, Westdeutschland

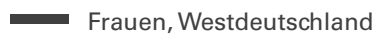

- Männer, Ostdeutschland

Frauen, Ostdeutschland

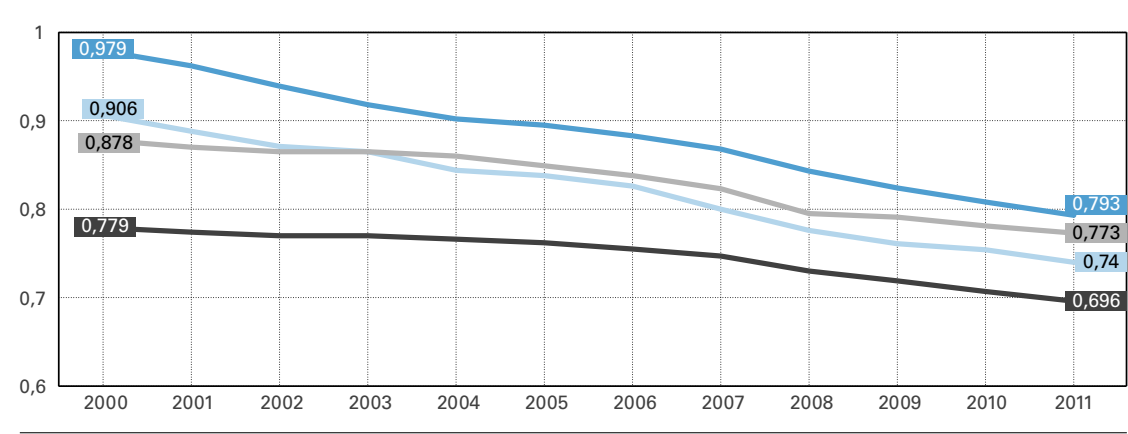

*Männer und Frauen in West- und Ostdeutschland.

Quelle: DRV 2012; Berechnungen und Darstellung des Autors.

Mitteilungen

ABB. 4

\section{Erwerbsminderungsrenten: Versicherungsstatus am 31.12. im Jahr vor dem Leistungsfall 2000 - 2012}

Angaben in Prozent aller Neuzugänge

Versicherungspflichtige Beschäftigung

Leistungsempfang nach SGB III)

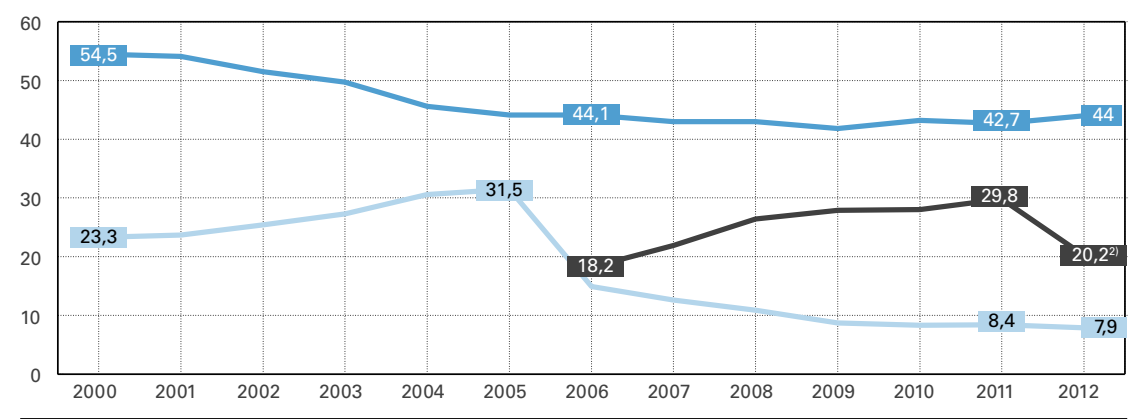

1) bis 2005 einschl. Arbeitslosenhilfe. 2) Hinzu gerechnet werden der Großteil der sog. Anrechnungszeitversicherten, da Zeiten des ALG II-Bezugs seit 2011 keine Beitrags-, sondern nur noch Anrechnungszeiten sind. Die restlichen Zugänge kommen aus sonstigen aktiven Versicherungsverhältnissen und aus einer passiven Versicherung.

Quelle: DRV 2013b; Berechnungen und Darstellung des Autors.

3 Ab 2005, mit der Einführung des SGB II, waren Zeiten des Bezugs von Arbeitslosengeld II Beitragszeiten, die zur Erfüllung der Wartezeiten genutzt werden konnten. Nach langjähriger Arbeitslosigkeit war es deshalb möglich, einen Anspruch auf den Bezug einer Erwerbsminderungsrente aufzubauen. Während dies für die Bezieher der vormaligen Arbeitslosenhilfe nach dem SGB III auch schon zuvor galt, stand diese Möglichkeit den vormaligen Empfängern von Sozialhilfe bis dato nicht offen. Es ist unbekannt, in welchem Ausmaß dadurch zusätzliche Ansprüche und auch Anträge auf Erwerbsminderungsrenten generiert 
ABB. 5

Erwerbsminderungsrenten: Durchschnittliche Zahlbeträge nach Versicherungsstatus vor Leistungsfall 2000 - 2012

Angaben in Euro

Versicherungspflichtige Beschäftigung

Leistungsempfang nach SGB III

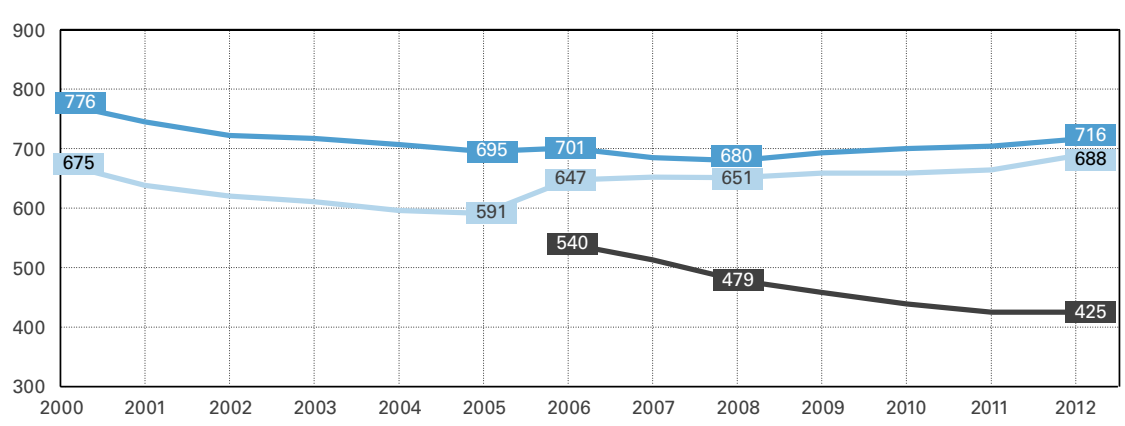

Quelle: DRV 2013b; Berechnungen und Darstellung des Autors. veau im Alter signalisieren, müssen demnach die in einem Haushalt womöglich anfallenden weiteren Einkommensarten wie Betriebsrenten, private Leibrenten, Wohngeld, Kapitaleinkünfte und auch Hinterbliebenenrenten addiert werden.

$\mathrm{Ob}$ ein niedriges Haushaltseinkommen im Alter das Kriterium „Armut“" erfüllt, hängt entscheidend von der Festlegung der Armutsschwelle ab. Verwendet man das politisch-institutionell festgelegte Bedarfsniveau der Grundsicherung im Alter und bei Erwerbsminderung (SGB XII) als Maßstab, so zeigt sich, dass sich nahezu die Hälfte der Leistungsempfänger (48,3 \% Ende 2011) aus dauerhaft Erwerbsgeminderten zusammensetzt, also aus Hilfebedürftigen, die zwischen 18 und 65 Jahren alt sind. Seit 2003 hat sich die Empfängerzahl von Leistungen der Grundsicherung insgesamt nahezu verdoppelt (von 439.000 auf 844.000). Zugleich ist der Anteil der Erwerbsgeminderten kontinuierlich gestiegen (Statistisches Bundesamt 2013).

Bezogen auf alle (inländischen) Erwerbsminderungsrentner im Alter bis zu 65 Jahren berechnet sich der Anteil derer, die Grundsicherungsleistungen in Anspruch nehmen müssen, auf 8,8 \% (10,6 \% bei den Männern und 8,4 \% bei den Frauen). Davon haben allerdings nur $27 \%$ überhaupt einen Rentenanspruch, die anderen waren entweder nie sozialversicherungspflichtig erwerbstätig (z. B. Schwerstbehinderte) oder erfüllen nicht die versicherungsrechtlichen Voraussetzungen für einen Rentenbezug. Zum Vergleich: Von den Altersrentnern sind es etwa 1,9\%, die eine aufstockende Grundsicherung beziehen.

Die Befunde aus der Grundsicherungsstatistik unterschätzen dabei noch die Betroffenheit. Denn die Grundsicherung im Alter und bei Erwerbsminderung begrenzt sich auf dauerhaft voll Erwerbsgeminderte. Zeitrentner sowie "nur“ teilweise Erwerbsgeminderte haben keinen Anspruch. Teilweise Erwerbsgeminderte werden auf das SGB II verwiesen, Zeitrentner (Vollrentner) auf die Sozialhilfe. Beide Gruppen tauchen deshalb in den genannten Zahlen nicht auf.

Unter den Leistungsempfängern im SGB II befinden sich aber nicht nur bedürftige Teil-Erwerbsgeminderte, sondern auch viele Langzeitarbeitslose, die wegen erheblicher gesundheitlicher Beeinträchtigungen zwar keine realistischen Chancen auf einen beruflichen Wiedereinstieg haben, wegen der weiten Definition von „Erwerbsfähigkeit“ oder wegen Nichterfüllung der Warte- und Pflichtbeitragszeit jedoch keine Erwerbsminderungsrente erhalten. Die Empfänger von Arbeitslosengeld II können auch keine Leistungsansprüche auf Erwerbsminderungsrenten mehr erwerben bzw. aufbauen, da es sich nicht mehr um Beitragszeiten handelt.

worden sind. Da seit 2011 für Empfänger von ALG II keine Rentenversicherungsbeiträge mehr gezahlt werden, ist diese Möglichkeit entfallen. Seitdem gelten Zeiten im ALG-II-Bezug als Anrechnungszeiten.
Niedrige Erwerbsminderungsrenten gehen nicht automatisch mit Armut einher. In der Armutsforschung ist unstrittig, dass zur Bestimmung von Einkommensarmut das verfügbare und nach Bedarf gewichtete Pro-Kopf-Haushaltseinkommen als Maßstab dient. Um zu überprüfen, ob niedrige Renten tatsächlich ein niedriges Einkommensni- 
Der Kenntnisstand über die sozioökonomische Lage von Erwerbsgeminderten bzw. von Personen, die im Haushalt mit Erwerbsgeminderten zusammenleben, ist bislang denkbar gering. Die Ergebnisse des von Mitarbeitern der Deutschen Rentenversicherung durchgeführten empirischen Forschungsprojektes (Märtin et al. 2012) führen deshalb zu wertvollen Erkenntnissen: Nach dem Konzept der aus der Einkommensverteilung abgeleiteten Armutsberechnung (als Armutsschwelle dienen hier $60 \%$ des durchschnittlichen (Median)Nettoäquivalenzeinkommens der Gesamtbevölkerung) können 36,5 \% aller Personen in den Haushalten der im Jahr 2010 Befragten als armutsgefährdet gelten. Wie zu erwarten, liegt die Armutsgefährdungsquote in Einpersonenhaushalten mit 49,6 \% dabei besonders hoch, da es keine Kompensation einer Niedrigrente durch das Einkommen des anderen Haushaltsmitglieds (in der Regel Ehepartner) gibt. Aber auch in den Einpersonenhaushalten ist die Erwerbsminderungsrente nicht in allen Fällen das einzige Einkommen. Vor allem die Hinterbliebenenrenten, aber auch Betriebsrenten haben hier eine Bedeutung.

Aufstockende Leistungen der Grundsicherung einschließlich Kosten der Unterkunft erhalten $18 \%$ der Personen in den Haushalten der befragten Erwerbsminderungsrentner. Auch hier liegt die Quote in Einpersonenhaushalten mit 27,9 \% merklich höher als in Mehrpersonenhaushalten mit 16,3\%. Insgesamt wird damit bei dieser Befragung eine um 9,2 Prozentpunkte höhere Grundsicherungsquote ermittelt als dies die Daten des Statistischen Bundesamtes ausweisen. Die Ursachen für diese erhebliche Abweichung sind nicht klar, ein möglicher Grund kann sein, dass - wie oben skizziert - viele Erwerbsgeminderte und ihre Angehörigen Grundsicherungsleistungen nach dem SGB II beziehen.

Eklatant sind Unterschiede zwischen der Armutsgefährdungsquote (36,5\%) und der Grundsicherungsquote (12,8 \%). Zu beachten bleibt dabei, dass sich die Armutsschwellen, die aus der Einkommensverteilung abgeleitet werden, nicht mit denen der Grundsicherung vergleichen lassen (Bäcker/ Schmitz 2012). Das Bedarfsniveau der Grundsicherung liegt in der Regel unter der relativen Armutsgrenze (60\% des Medians), aber da es sich nicht um eine exakte Grenze handelt, sondern um haushalts- und lebenslagenbezogene Beträge (z.B. Zuschläge für Behinderte oder besonders hohe Mieten), kann es auch sein, dass die Grundsicherung höher liegt. Auch ist bei dem Vergleich der beiden Armutskonzepte immer in Rechnung zu stellen, dass die Grundsicherungs- als Prozessstatistik naturgemäß nur jene Personen erfasst, die einen Antrag stellen und diesen bewilligt erhalten. Es ist aber bekannt, dass ein erheblicher Teil der Bezugsberechtigten von dem Recht auf aufstockende Grundsicherungsleistungen keinen Gebrauch macht (Becker 2012). Aus der Befragung lässt sich für 23,7 \% feststellen, dass sie zwar armutsgefährdet sind, aber keine Grundsicherungsleistungen erhalten. 5,2 \% geben an, nicht armutsgefährdet zu sein, aber im Grundsicherungsbezug zu stehen.
ABB. 6

\section{Zugänge in Alters- und Erwerbsminderungsrenten und Anteile von EM-Renten 1995 - 2012}

Angaben in Euro und Prozent

Altersrenten $\quad$ Erwerbsminderungsrenten
Anteil der Erwerbsminderungsrenten am Rentenzugang insgesamt in \%

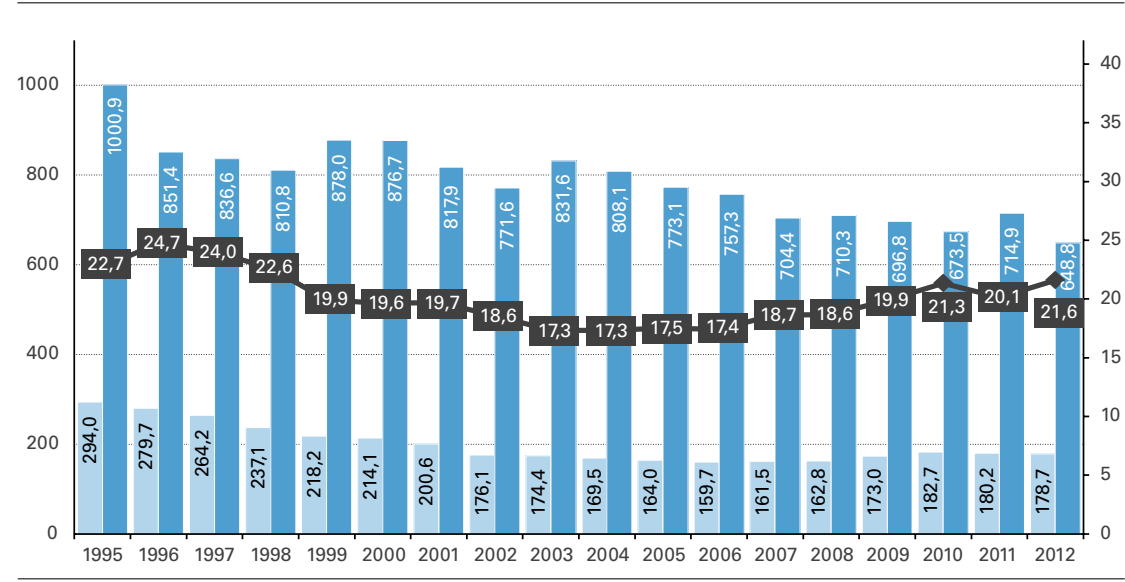

Quelle: DRV ; Berechnungen und Darstellung des Autors.

\section{Ein Blick in die Zukunft}

Seit etwa 2004/2005 nimmt die Bedeutung der Erwerbsminderungsrenten am gesamten Rentenzugang $\mathrm{zu}^{4}$ (Abbildung 6). Und es spricht viel dafür, dass sich dieser Trend fortsetzen wird. Denn die seit Anfang 2012 in Kraft getretene stufenweise Heraufsetzung der Regelaltersgrenze auf 67 Jahre und die schon seit längerer Zeit wirksamen Schritte der Heraufsetzung der vorgezogenen Altersgrenzen bis hin zur Abschaffung der vorgezogenen Altersrente für Frauen wegen Arbeitslosigkeit und nach Altersteilzeit ( $a b$ 2012, jeweils für Geburtsjahrgänge ab 1952) führen dazu, dass sich die Möglichkeiten eines frühzeitigen Altersübergangs durch Bezug einer Altersrente - auch um den Preis von Abschlägen - zunehmend beschränken. In der Folge rücken vermehrt Versicherte, die 60 Jahre und älter sind, in den Kreis potenzieller Erwerbsminderungsrentner. Da im höheren Alter zugleich das Risiko eines Verlustes der Erwerbsfähigkeit aufgrund schwerer Erkrankungen steigt, spricht viel für die Erwartung, dass Erwerbsminderungsrenten in den nächsten Jahren vermehrt beantragt und (zum

4 Hier werden die Anteile an allen Rentenneuzugängen betrachtet. Demografische Einflüsse in Form von unterschiedlichen Besetzungsstärken der Jahrgänge bleiben dabei unberücksichtigt. Ausschalten lässt sich dies durch den Vergleich von Kohorten (Bäcker 2012) oder durch den Bezug auf aktiv Versicherte (Kaldybajewa/Kruse 2012). 
Teil) auch bewilligt werden. Die Einschränkung des Bezugs vorzeitiger Altersrenten müsste sich in einer wachsenden Bedeutung der Altersgruppe der 60-Jährigen und Älteren beim Zugangsgeschehen äußern. Tatsächlich zeigen die Daten der Rentenzugangsstatistik, dass die Altersgruppe 60 bis unter 65 Jahre beim Bezug einer Erwerbsminderungsrente an Gewicht gewinnt, allerdings ist der Anstieg noch schwach: bei den Männern von 8,2 \% im Jahr 2007 auf 13,1 \% im Jahr 2012, bei den Frauen von 4,7 auf 7,5\% (DRV 2013b).

Zugleich spricht wenig dafür, dass sich die Einkommenslage der Betroffenen grundlegend verbessern wird. Im Zuge der Anhebung der Regelaltersgrenze vergrößert sich vielmehr die Spanne zwischen dem Ende der Zurechnungszeit (60 Jahre) und der Regelaltersgrenze (67 Jahre im letzten Anhebungsschritt) auf sieben Jahre. Im Vergleich zu den Altersrenten (bei einem Renteneintritt ohne Abschläge) fallen dadurch die Erwerbsminderungsrenten zunehmend niedriger aus. Und die Absenkung des Rentenniveaus wird vor allem die Erwerbsminderungsrentner treffen. Denn es hat sich gezeigt, dass die Philosophie des Paradigmenwechsels in der Alterssicherung, nämlich die Rückführung des Leistungsniveaus der Rentenversicherung bei einem gleichzeitigen, durch Zulagen und Steuererleichterungen geförderten Aufbau der privaten und betrieblichen Altersvorsorge, bei den Erwerbsminderungsrenten nicht greift (Köhler-Rama et al. 2010). Denn es ist vor allem für Risikogruppen (gering Qualifizierte, Langzeit- und Mehrfacharbeitslose, schlechter Gesundheitszustand und Vorerkrankungen) (vgl. Promberger et al. 2012), die zugleich ein hohes Risiko des Eintritts einer Erwerbsminderung haben, nur begrenzt möglich, sich adäquat privat oder betrieblich gegen dieses Risiko abzusichern. Die privaten Versicherer bieten entsprechende Produkte selten an - und wenn, dann zu kaum bezahlbaren Tarifen. Vor allem bei einem Eintritt der Erwerbsminderung in jüngeren Jahren bestehen keine Möglichkeiten, ausreichend lange privat oder betrieblich vorzusorgen (Asshoff/Mathes 2009).

\section{Reformbedarfe}

Über den dringenden Reformbedarf bei den Erwerbsminderungsrenten gibt es bei den Experten keinen Dissens (vgl. u. a. Nürnberger 2009; Rische 2010; Bäcker et al. 2011; Rische/Kreikebohm 2012; Welti/Groskreutz 2013). Angesichts der zentralen Bedeutung, die das Rentenniveau für die Armutsfestigkeit der Renten allgemein und der Erwerbsminderungsrenten im Besonderen hat (vgl. Bäcker 2013), sollte dabei der Suche nach Wegen, das Rentenniveau zumindest auf dem aktuellen Stand zu stabilisieren, eine vorrangige Bedeutung zukommen. Ohne eine Veränderung der Rentenanpassungsformel ist dies nicht möglich.

Aber auch die Berechtigung von Abschlägen ist kritisch zu hinterfragen. Zwar verstößt ihre Einführung nach der Rechtsprechung des Bundesverfassungsgerichtes (Entscheidung vom 11.01.2011) nicht gegen das Grundgesetz. Dennoch ist diese Regelung für Erwerbsminderungsrenten nicht systemgerecht. Denn Abschläge beziehen sich in ihrer Logik auf Altersrenten und sind so bemessen, dass die mit einem vorgezogenen Beginn einer Altersrente einhergehende Verlängerung der Rentenbezugsdauer nicht zu einer finanziellen Mehrbelastung der Rentenversicherung führt. Zugleich sollen sie das Rentenzugangsverhalten steuern, indem sie spürbar werden lassen, dass es „teuer“ ist, frühzeitig eine Rente zu beziehen. Geht man von einem korrekten medizinischen Beurteilungsverfahren aus, dann können aber Erwerbsgeminderte ihren Gesundheitszustand nicht so weitgehend beeinflussen, dass sie wieder in der Lage sind, eine Arbeit aufzunehmen. Weder der Verlust der Erwerbsfähigkeit noch der Zeitpunkt des Renteneintritts sind freiwillig gewählt und deswegen mit der Inanspruchnahme einer vorgezogenen Altersrente nicht vergleichbar. Auch das Argument der Finanzneutralität bei einer verlängerten Rentenlaufzeit kann bei Erwerbsminderungsrenten nicht greifen, da die Erwerbsminderung nicht an eine Altersgrenze gebunden ist, sondern schon früh im Leben eintreten kann und - bei einer unterstellt gleichen durchschnittlichen Lebenserwartung wie von Altersrentnern - sehr viel länger gezahlt wird.

Eine Begrenzung der Abschläge ist deshalb zwingend geboten. Allerdings ist es für die Betroffenen nicht entscheidend, wie eine bessere finanzielle Absicherung beim Eintritt einer Erwerbsminderung erreicht wird. Richtig ausgestaltet lässt sich auch durch eine Verlängerung der Zurechnungszeiten eine analoge Erhöhung der Rentenzahlbeträge erreichen.

Es kommt aber auch darauf an, gerade im Bereich besonders niedriger Erwerbsminderungsrenten für einen Ausgleich zu sorgen und die hier niedrigen Entgeltpunkte anzuheben. Der Ansatz bei der Rentenberechnung, zukünftig eine Günstigerprüfung vorzunehmen und die letzten vier Jahre vor dem Eintritt der Erwerbsminderung nicht zu berücksichtigen, wenn sich dies nachteilig auf die Bewertung der Zurechnungszeiten auswirkt, ist ein Schritt in diese Richtung. Dessen Wirksamkeit hängt aber davon ab, in welcher Stärke sich die letzten vier Jahre negativ auswirken. Dies wäre empirisch zu überprüfen (zu ersten Ergebnissen vgl. Gasche/Härtl 2013). Schließlich stellt sich gerade für Erwerbsminderungsrentner die Frage, wie Zeiten der Arbeitslosigkeit im SGB II besser bewertet werden können (Steffen 2011).

\section{LITERATUR}

Asshoff, G./Mathes, M. (2009): Möglichkeiten und Grenzen eines Ausbaus der betrieblichen und privaten Absicherung, in: Soziale Sicherheit 58 (9), S. 306-311

Bäcker, G. (2012): Erwerbsminderungsrenten: Strukturen, Trends und aktuelle Probleme: Institut Arbeit und Qualifikation, Altersübergangs-Report (3), Duisburg

Bäcker, G. (2013): Niedrigrenten, Arbeitsmarkt und Rentenversicherung - Erfordernis einer integrierten Ursachenanalyse und Reformstrategie, in: Kistler, E./Trischler, F./Voss, D. (Hrsg.): Reformen auf dem Arbeitsmarkt und in der Alterssicherung - Folgen für die Einkommenslagen im Alter, Arbeitspapiere der Hans-Böckler-Stiftung, Düsseldorf (im Erscheinen)

Bäcker, G./Kistler, E./Stapf-Finé, H. (2011): Erwerbsminderungsrente - Reform notwendigkeiten und Reformoptionen: Friedrich-Ebert-Stiftung, WISO-Diskurs Bonn

Bäcker, G./Schmitz, J (2012): Altersarmut und Rentenversicherung: Diagnosen, Trends, Reformoptionen und Wirkungen, in: Vogel, C./Motel-Klingebiel, A. (Hrsg.): Altern im sozialen Wandel. Die Rückkehr der Altersarmut, Wiesbaden, S. $25-56$

Becker, I. (2012): Finanzielle Mindestsicherung und Bedürftigkeit im Alter, in Zeitschrift für Sozialreform 58 (2), S. 123-148

Deutsche Rentenversicherung Bund (DRV) (2012): Rentenversicherung in Zeitreihen, Berlin

Deutsche Rentenversicherung Bund (DRV) (2013a): Rentenversicherung in Zahlen, Berlin

Deutsche Rentenversicherung Bund (DRV) (2013b): Rentenzugang 2012 und ältere Jahrgänge, Berlin

Deutscher Bundestag (2000): Entwurf eines Gesetzes zur Reform der Renten 
wegen verminderter Erwerbsfähigkeit, Bundestagsdrucksache 14/4230 Frommert, D./Himmelreicher, R. (2010): Sinkende Rentenanwartschaften - vor allem in den neuen Bundesländern, in: Informationsdienst Soziale Indikatoren (43), S. $1-5$

Gasche, M./Härtl, K. (2013): Verminderung der (Alters-)Armut von Erwerbsminderungsrentnern durch Verlängerung der Zurechnungszeit und Günstigerprüfung?, Max-Planck-Institut für Sozialrecht und Sozialpolitik, mea discussion papers (217), München

Hagen, C./Himmelreicher, R./Kemptner, D./Lampert, T. (2011): Soziale Ungleichheit und Risiken der Erwerbsminderung, in: WSI-Mitteilungen 64 (7), S. 336-344, http://www.boeckler.de/wsi_36800_36908.htm

Kaldybajewa, K./Kruse, E. (2012): Erwerbsminderungsrenten im Spiegel der Statistik der gesetzlichen Rentenversicherung - Unterschiede und Gemeinsamkeiten zwischen Männern und Frauen, in: RV-aktuell (9), S. 201-216

Köhler-Rama, T./Lohmann, A./Viebrock, H. (2010): Vorschläge zu einer Leistungsverbesserung bei Erwerbsminderungsrenten aus der gesetzlichen Rentenversicherung, in: Zeitschrift für Sozialreform 56 (1), S. 59-83

Märtin, S./Zollmann, P./Buschmann-Steinhage, R. (2012): Sozioökonomische Situation von Personen mit Erwerbsminderung, DRV-Schriften (99), Berlin Mika, T. (2013): Risiken für eine Erwerbsminderung bei unterschiedlichen Berufsgruppen, in: Bundesgesundheitsblatt (3), S. 391-398

Nürnberger, I. (2009): Erwerbsgeminderte besser absichern!, in: Soziale Sicherheit 58 (3), S. 85-92

Promberger, M./Wübbeke, C./Zylowski, A. (2012): Arbeitslosengeld-II-Empfänger: Private Altersvorsorge fehlt, wo sie am nötigsten ist, IAB-Kurzmitteilungen 15/2012, Nürnberg

Rehfeld, U. (2006): Gesundheitsbedingte Frühberentung: Robert Koch-Institut, Gesundheitsberichterstattung des Bundes (30), Berlin

Rische, H. (2010): Die Absicherung des Erwerbsminderungsrisikos - Handlungsbedarf und Reformoptionen, in: RV aktuell (1), S. 1-9

Rische, H./Kreikebohm, R. (2012): Verbesserung der Absicherung bei Invalidität und mehr Flexibilität beim Übergang in Rente, in: RV aktuell (1), S. 2-16
Statistisches Bundesamt (2013): Empfänger und Empfängerinnen von Grundsicherung im Alter und bei Erwerbsminderung, Fachserie 13, Reihe 2.2., Wiesbaden

Steffen, J. (2011): Arbeitslosigkeit und Rente. Modelle zur Absicherung von Zeiten der Arbeitslosigkeit in der gesetzlichen Rentenversicherung, http:// www.sozialpolitik-aktuell.de/tl_files/sozialpolitik-aktuell/_Politikfelder/AlterRente/Dokumente/2011-03-02\%20Arbeitslosigkeit\%20und\%20Rente.pdf Steffen, J. (2013): Erwerbsminderungsrenten im Sinkflug - Ursachen und Handlungsoptionen, http://www.sozialpolitik-portal.de/uploads/sopo/ pdf/2013/2013-05-27-Erwerbsminde-rungsrenten_im_Sinkflug_PS.pdf Trischler, F. (2012): Auswirkungen diskontinuierlicher Erwerbsbiografien auf die Rentenanwartschaften, in: WSI-Mitteilungen 65 (4), S. 253-261, http://www.boeckler.de/wsimit_2012_04_trischler.pdf

Weber, B./Weber, E. (2013): Qualifikation und Arbeitsmarkt - Bildung ist der beste Schutz vor Arbeitslosigkeit, in: IAB-Kurzbericht 4/2013, Nürnberg Welti, F./Groskreutz, H. (2013): Vorschlag für eine grundlegende Reform im Erwerbsminderungsrecht, in: Soziale Sicherheit 62 (8-9), S. 308-311

\section{AUTOR}

GERHARD BÄCKER, Prof. Dr., ist als Senior-Professor am Institut Arbeit und Qualifikation (IAQ) der Universität Duisburg-Essen tätig. Arbeitsschwerpunkte: Soziologie des Sozialstaates, insbesondere Alterssicherung, Arbeitsmarkt, Armut.

gerhard.baecker@uni-due.de 